\title{
Semantics for Counterpossibles
}

\author{
Yale Weiss \\ The Graduate Center, CUNY \\ yweiss@gradcenter.cuny.edu
}

\begin{abstract}
The object of this paper is to examine two approaches to giving non-vacuous truth conditions for counterpossibles, counterfactuals with impossible antecedents. I first develop modifications of a Lewis-style sphere semantics with impossible worlds. I argue that this approach sanctions intuitively invalid inferences and is supported by philosophically problematic foundations. I then develop modifications of certain ceteris paribus conditional logics with impossible worlds. Tableaux are given for each of these in an appendix and soundness and completeness results are proved. While certain of the latter systems are shown to have similar problems to logics from the first approach, at least one relatively weak system appears to offer an adequate uniform semantics for counterpossibles and counterfactuals.
\end{abstract}

\section{Introduction}

Lewis [1] developed a robust account of would and might counterfactuals, analyzing them as variably strict conditionals. His account has many merits, but shares with [18] the problem of making all counterpossibles, counterfactuals with impossible antecedents, vacuously true. That this is a problem can be motivated with a simple example. The conditional "if there were a counterexample to the law of the excluded middle, Brouwer would have been right not to trust that law" seems to be true whereas "if there were a counterexample to the law of the excluded middle, Brouwer would have been wrong not to trust that law" seems to be false. I will take the unacceptability of this for granted in what follows. 17

\footnotetext{
${ }^{1}$ For a recent survey of arguments against vacuism, see [2].
} 
One approach to rectifying this problem is to simply naively extend Lewis' system to encompass impossible worlds as well as possible ones.$^{2}$ Let us write $A \square \mapsto B$ for "if it were the case that $A$, it would be the case that $B$ " and call a world at which $A$ is true an $A$-world. Then, jettisoning the vacuous case, the truth conditions for would counterfactuals (informally) become,

(T) $A \square \rightarrow B$ is true at world @ if some $A$-worlds which are $B$ worlds, possible or not, are closer to @ than any $A$-worlds which are not $B$-worlds, possible or not (cf. [19, p. 188])

To evaluate "if there were a counterexample to the law of the excluded middle, Brouwer would have been right to mistrust that law", we look at worlds close (similar) to the actual world in which there are counterexamples to this law (which are, ex hypothesi, impossible worlds). Of these, those where Brouwer holds the same opinions about the law of the excluded middle that he actually does hold are closer to the actual world than any worlds in which he holds different views. So the counterpossible comes out non-vacuously true on the condition given in (T).

Lewis articulates the crucial notion of similarity using an apparatus of nested spheres. In the first section, I develop several versions of a Lewis-style sphere semantics with impossible worlds to accommodate (T). I argue that this approach is philosophically problematic and sanctions as valid certain intuitively invalid inferences.

In the second section, I turn to a different approach to giving semantics for counterfactuals developed by Chellas in [3]- [4] and Priest in [15]-[17]. I develop several versions of this semantics with impossible worlds (sound and complete tableaux for these are provided in an appendix). I argue for the superiority of this approach by indicating how at least one of these systems avoids the more serious problems of the Lewisian approach while also meeting certain minimum adequacy conditions.

\section{Sphere Semantics}

\subsection{Language and Semantics}

I present a fairly general version of Lewis' sphere semantics extended with impossible worlds below. To expedite things, I build this logic on top of a basic propositional modal language.

\footnotetext{
${ }^{2}$ The logics developed below implicitly characterize what impossible worlds are, but questions about their ontological status are left aside. For a recent discussion of ontological issues, see 10 .
}

Australasian Journal of Logic (14:4) 2017, Article no. 1 
DEFINITION. The set of formulae are defined by the following recursive clauses: 3

1. Every propositional letter is a formula (assume we have countably many: $\left.P, P_{0}, P_{1}, \ldots Q, \ldots\right)$

2. If $\phi$ is a formula, so are $\neg \phi, \diamond \phi$ and $\square \phi$

3. If $\phi$ and $\psi$ are formulae, so are $(\phi \vee \psi),(\phi \wedge \psi),(\phi \rightarrow \psi)$ and $(\phi \square \rightarrow \psi)$

DEFINITION. An interpretation is a structure $\left\langle W, N,\left\{\$_{i}: i \in N\right\}, \rho\right\rangle$, where $W$ is a set of worlds and $\emptyset \neq N \subseteq W$ is the set of normal worlds ( $W-N$ is the set of non-normal worlds). $\$_{i}$ is a set of subsets of $W,\left\{S_{0}, S_{1}, \ldots, S_{n}\right\}$, such that $S_{0} \subseteq S_{1} \subseteq S_{2} \subseteq \ldots \subseteq S_{n}=W \bigsqcup^{5}$ For all $w \in W, \rho_{w}$ is a relation between propositional letters and $\{0,1\}$. Moreover, for all $w \in W-N, \rho_{w}$ is a relation between formulae generally and $\{0,1\}$. Finally, for all propositional letters $\phi$ and $w \in N$, either $\phi \rho_{w} 1$ or $\phi \rho_{w} 0$, but not both. 1 is the designated value ("true").

Lewis calls the elements of $\$_{i}$ "spheres," and his intuition is that smaller spheres contain worlds which are more similar overall to world $i$ than those found only in larger spheres, and this is supposed to be relevant to the assessment of counterfactuals at (normal) worlds. Note that all formulae at non-normal worlds are evaluated directly on the semantics offered here. Thus, $\$_{i}$ does not enter into the truth conditions for counterfactuals at nonnormal worlds.

Because $\rho_{w}$ is an arbitrary relation for $w \in W-N$, it can associate a formula with both, neither or one of $\{0,1\}$. Therefore, not every world is maximal in the sense that there can be worlds at which not every formula receives a truth value. The inclusion of such "partial" worlds in the models is desirable for several reasons which I will not review here (for some unintuitive consequences which result when partial worlds are not included, see [1]).

DEFINITION. Define $[\phi]$ to be $\left\{x \in W: \phi \rho_{x} 1\right\}$. Truth conditions for complex formulae at normal worlds $(w \in N)$ are given by the following recursive clauses:

\footnotetext{
${ }^{3}$ Following convention, I will typically omit outer parentheses. $\phi \leftrightarrow \psi$ abbreviates $(\phi \rightarrow \psi) \wedge(\psi \rightarrow \phi)$.

${ }^{4}$ It is assumed, for simplicity, that all $\$_{i}$ have finite cardinality. This implies the Limit Assumption: whenever there is a $\phi$-world, there is at least one closest $\phi$-world. For the reasons given in [11, pp. 19-20], [12, pp. 229-30] and [2, pp. 640-1], the Limit Assumption ought not to be made. Problems with the Limit Assumption are entirely orthogonal to the subject of this paper, however, and will be ignored accordingly.

${ }^{5}$ I.e. $\$_{i}$ is nested, closed under unions and non-empty intersections and universal [11, pp. 13-6].
}

Australasian Journal of Logic (14:4) 2017, Article no. 1 
1. $\neg \phi \rho_{w} 1$ if and only if $\phi \rho_{w} 0 ; 0$ otherwise

2. $(\phi \wedge \psi) \rho_{w} 1$ if and only if $\phi \rho_{w} 1$ and $\psi \rho_{w} 1 ; 0$ otherwise

3. $(\phi \rightarrow \psi) \rho_{w} 1$ if and only if $\phi \rho_{w} 0$ or $\psi \rho_{w} 1 ; 0$ otherwise

4. $(\phi \vee \psi) \rho_{w} 1$ if and only if $\phi \rho_{w} 1$ or $\psi \rho_{w} 1 ; 0$ otherwise

5. $\square \phi \rho_{w} 1$ if and only if $N \subseteq[\phi] ; 0$ otherwise

6. $\diamond \phi \rho_{w} 1$ if and only if $N \cap[\phi] \neq \emptyset ; 0$ otherwise

7. $(\phi \square \hookrightarrow \psi) \rho_{w} 1$ if and only if $\exists S \in \$_{w}$ such that $\emptyset \neq[\phi] \cap S \subseteq[\psi] ; 0$ otherwise

Validity is defined as truth preservation at all normal worlds of all interpretations. Call this logic $S^{\#}$. The similarity spheres picture can be used to motivate a variety of additional constraints on $\$_{i}$. On the grounds that no world is as similar to a given world $i$ than $i$ itself, Lewis advocated for ${ }^{6}$

$$
\text { (SC) }\{i\}=S_{0}
$$

Unfortunately, (SC) licenses the problematic inference $\phi, \psi \models(\phi \square \leftrightarrow \psi) !^{7}$ Suppose that Fred is a wealthy speculator who invested millions of dollars in pounds just before the Brexit vote. In spite of his significant loss the next morning, Fred is still quite rich. Then "Fred invested in the pound just before Brexit" is true and "Fred is quite rich" is true. But the conditional "if it were the case that Fred invested in the pound just before Brexit, then it would be the case that Fred is quite rich" does not seem to be true. More modestly, it might be required that,

$$
\text { (WC) } i \in S_{0}
$$

To round out the semantics, constraints on the models pertaining to impossible worlds must be considered.

Nothing in the foregoing requires that every formula $\phi$ be satisfied at some world. In models where certain formulae are not true at any worlds, possible or not, counterfactuals with those formulae as antecedents will be false when evaluated from a possible world. Alternatively, following [11], the truth conditions could be recast so that when $\phi$ is not true at any worlds, counterfactuals with it as an antecedent are true at possible worlds. In practice, it makes little difference whether such counterfactuals are rendered

\footnotetext{
${ }^{6}$ In Lewis' terminology, $\$_{i}$ is "centered on $i$ " [11, p. 14].

${ }^{7}$ Problems with this inference are noted in [8] and [15, p. 96].
} 
true or false since there are models in which every formula is true at some world. For variety, I treat such counterfactuals as true in the alternative semantics presented in the following section.

If it is thought to be desirable to ensure that, in all models, every formula is satisfied at some world, a trivial way to do this is to require the existence, in every model, of an impossible world which satisfies every formula. Call this trivial world $\lambda$ (cf. [18, p. 46]). Even if it is not required that $\lambda$ exist in all models, its existing in some models neither is nor should be proscribed. It is as legitimate an impossible world as any other.

How distant should impossible worlds, both extreme (such as the trivial world) and more mundane, be from a given possible world? It is perhaps natural to suppose that all possible worlds are more similar overall to a given possible world than any impossible worlds. This gives rise to a condition which Nolan calls "strangeness of impossibility" [13, p. 550], and I will refer to as,

(Dis) $N \in \$_{i}$

Similarly, it is plausible to require that the trivial world (if it exists) occur only in the most distant sphere (cf. [13, p. 544], [2, p. 652]). That is,

$$
\text { (Ab) } \forall k<n, S_{k} \cap\{\lambda\}=\emptyset
$$

The following extensions of $S^{\#}$ should be noted:

$$
\begin{aligned}
& S_{1}^{\#}: S^{\#}+(\mathrm{WC}) \\
& S_{2}^{\#}: S^{\#}+(\mathrm{SC}) \\
& S_{3}^{\#}: S^{\#}+(\mathrm{WC})+(\mathrm{Dis})+(\mathrm{Ab})
\end{aligned}
$$

$S_{1}^{\#}$ is the counterpossible correlate of the logic Priest calls S 15, pp. 902]. $S_{2}^{\#}$ is the most distinctly Lewisian counterpossible logic. $S_{3}^{\#}$ is, among counterpossible sphere logics, the one which I take to most nearly capture prevalent intuitions about the nearness of impossible worlds. In the following section, I direct criticisms at each of these.

\subsection{Formal and Philosophical Issues}

While many inference patterns which are valid in Lewis' systems fail to be valid in $S^{\#}$ and its extensions, they frequently have modal parallels in $S_{3}^{\#}$ which hinge crucially on (Dis). Two, in particular, should be noted:

R1) $\square \phi, \square \psi \models_{S_{3}^{\#}} \phi \square \rightarrow \psi$

Australasian Journal of Logic (14:4) 2017, Article no. 1 
R2) $\diamond \phi, \square(\phi \rightarrow \psi) \models{ }_{S_{3}^{\#}} \phi \square \mapsto \psi$

(R1) corresponds to the inference sanctioned by (SC) in traditional Lewis semantics and $S_{2}^{\#}$ (noted above). Consider an arbitrary world $w \in N$ of an arbitrary model such that $\square \phi \rho_{w} 1$ and $\square \psi \rho_{w} 1$. Since $w \in N \subseteq[\phi]$ and $N \subseteq[\psi], \emptyset \neq[\phi] \cap N \subseteq[\psi]$. By (Dis) $\exists S=N \in \$_{w}$. Therefore, $(\phi \square \leftrightarrow \psi) \rho_{w} 1$.

The counterpart of (R2) in Lewis' preferred system is $\square(\phi \rightarrow \psi) \models \phi \square \rightarrow$ $\psi$. This rule encapsulates the relationship between strict and variably strict conditionals. To construct a countermodel, just let the strict conditional hold vacuously at some possible world and let the counterfactual fail because some sphere contains impossible antecedent-worlds which do not satisfy the consequent. (R2) is valid in $S_{3}^{\#}$ though. Consider an arbitrary world $w \in N$ of an arbitrary model such that $\diamond \phi \rho_{w} 1$ and $\square(\phi \rightarrow \psi) \rho_{w} 1$. Then $\emptyset \neq[\phi] \cap$ $N \subseteq[\psi]$. Since by (Dis) $\exists S=N \in \$_{w},(\phi \square \mapsto \psi) \rho_{w} 1$.

These inferences are problematic, however. Consider (R1). Let $\phi$ be "there is a counterexample to the law of the excluded middle or there is not a counterexample to the law of the excluded middle" and $\psi$ be "there is not a counterexample to the law of the excluded middle." Since the law of the excluded middle is valid (ex hypothesi), both of the premises, $\square \phi$ and $\square \psi$, are true. But the conclusion, "if it were the case that there is a counterexample to the law of the excluded middle or there is not a counterexample to the law of the excluded middle, it would be the case that there is not a counterexample to the law of the excluded middle," seems to be false. In evaluating this conditional, we should take into consideration worlds where each disjunct holds, i.e. both possible and impossible worlds. This is precisely what (Dis) rules out 8

The philosophical justification of (Dis) ought to be carefully scrutinized. Let us say that a logic $\mathcal{L}$ holds at (or governs) a world if the world is closed under the consequence relation of $\mathcal{L}$. For example, classical $\mathrm{S} 5$ holds at all of the possible worlds in $S^{\#}$ and its extensions. Among the worlds at which classical logic holds are many quite bizarre ones. It does not seem entirely obvious that strange but classically governed worlds are more similar to the actual world than worlds which are pretty much the same except that some paraconsistent logic holds. That is, (Dis) seems to depend on a prioritization of the laws of logic in judgments of similarity that is itself dubious.

In fact, such a prioritization creates even greater problems for $S_{3}^{\#}$ when $(\mathrm{Ab})$ is considered. I have suggested that the case for (Dis) rests on some

\footnotetext{
${ }^{8}(\mathrm{R} 1)$ is also open to objections of relevance. For example, take $\phi$ to be some banal instance of the law of the excluded middle ("there is a red thing or there is not a red thing") and $\psi$ to be the fundamental theorem of arithmetic. I will not be advocating for a relevance logic in this paper and intend to bracket such considerations.
} 
argument which prioritizes logical laws to, among other things, physical laws and chance occurrences. (Dis) seems to require that the most important consideration in gauging overall similarity is what logic holds at a world. The very same considerations militate against (Ab), for among the impossible worlds (or, at least, the inconsistent worlds), the trivial world, $\lambda$, is the one whose logical structure is the most like that of a possible world: $\lambda$ is the only inconsistent world closed under classical S5. Thus, the justification of (Dis) not only contravenes $(\mathrm{Ab})$, but suggests that $\lambda$ be among the "nearest" impossible worlds with the result that counterpossibles end up vacuously true again.

Of course, one need not take prioritization of logical laws as gospel in assessments of similarity. The challenge to those who do not is to produce some other justification of (Dis) and argument for (Ab). While I believe that most authors who have endorsed (Dis) do have some justification along these lines in mind, I do not claim that no other justification is possible, nor will I try to give one. I have already argued that (Dis) licenses intuitively invalid inferences, so if it lacks justification as well, so much the worse for it and, by extension, $S_{3}^{\#}$.

Since I have already argued against (SC) and $S_{2}^{\#}$, let us turn to $S_{1}^{\#}$. Three interesting inference patterns that hold due to (WC) in $S_{1}^{\#}$ and its extensions are the following: 9

$$
\begin{aligned}
& \text { R3) } \phi, \neg \psi \models_{S_{1}^{\#}} \neg(\phi \square \mapsto \psi) \\
& \text { R4) } \phi, \phi \square \mapsto \psi \models_{S_{1}^{\#}} \psi \\
& \text { R5) } \neg \psi, \phi \square \rightarrow \psi \models_{S_{1}^{\#}} \neg \phi
\end{aligned}
$$

For (R3), consider an arbitrary world $w \in N$ of an arbitrary model such that $\phi \rho_{w} 1$ and $\neg \psi \rho_{w} 1$. Then by (WC), $w \in[\phi] \cap S_{0} \nsubseteq[\psi]$ since $\psi \rho_{w} 0$. As

\footnotetext{
${ }^{9}$ Lewis endorses the Duality Thesis, (DT), which concerns the relationship between the conditionals $\square \rightarrow$ and $\diamond \rightarrow$ ("if it were,... it might be...") [11, p. 2]. Under (DT), $\phi \square \leftrightarrow \psi \equiv \neg(\phi \diamond \neg \neg \psi)$ and so (R3) can be rewritten: $\phi, \neg \psi \models \phi \diamond \neg \neg \psi$. But (DT) cannot be allowed to hold in $S^{\#}$ or its extensions. Absent the vacuous-truth case in the truth conditions for $\square \rightarrow$ conditionals, it must be the case that $\phi \square \rightarrow \psi \models \phi \diamond \psi$. Then there are models in which $\exists S \in \$_{w}$ such that $\emptyset \neq S \cap[\phi \wedge \neg \phi] \subseteq[\phi] \cap[\neg \phi]$. Then $(\phi \wedge \neg \phi \square \rightarrow \phi) \equiv \neg(\phi \wedge \neg \phi \diamond \neg \neg) \rho_{w} 1 .(\phi \wedge \neg \phi \square \mapsto \neg \phi) \rho_{w} 1$ implies $(\phi \wedge \neg \phi \diamond \neg \neg \phi) \rho_{w} 1$. So $w$, a possible world, satisfies a contradiction. Less formally, it is easy to appreciate that (DT) is intuitively wrong. "If it were the case that cats were and were not mammals, it would be the case that cats were mammals" seems true. Similarly, "if it were the case that cats were and were not mammals, it might be the case that cats were not mammals" seems true. But (DT) requires the negation of the latter to be true if the first is true. For other issues with (DT), see [5] and [6].
}

Australasian Journal of Logic (14:4) 2017, Article no. 1 
$\nexists S \in \$_{w}$ such that $\emptyset \neq[\phi] \cap S \subseteq[\psi],(\phi \square \rightarrow \psi) \rho_{w} 0$. Thus, $\neg(\phi \square \mapsto \psi) \rho_{w} 1$. The proofs of (R4) and (R5), modus ponens and modus tollens for $\square \rightarrow$, proceed similarly.

It is natural to take (R3) through (R5) as inferences that should hold in any minimally adequate logic for counterfactuals and counterpossibles. Indeed, they seem to be inferences that should hold in any theory of conditionals at all. Since each depends on (WC), it is worth investigating what the nested sphere structure on its own validates, i.e. what inferences involving conditionals are valid in $S^{\#}$. Among these is:

$$
\text { R6) } \phi \square \rightarrow \psi, \psi \square \rightarrow \phi \models_{S_{\#}}(\phi \square \hookrightarrow \chi) \leftrightarrow(\psi \square \leftrightarrow \chi)
$$

(R6), in its axiomatic guise, is widely known in the literature as (CSO) [14, p. 160]. Given that it only crucially depends on the nesting of spheres, it holds in $S^{\#}$ and all of its extensions ${ }^{10}$ Consequently, any objection to (R6) is an objection to sphere semantics in general. Consider the following scenario ${ }^{11}$ Fred is teaching George arithmetic. Fred asks George what $5+7$ is, and George mistakenly responds 13 . Fred snidely remarks, "if $5+7$ were 13, you would have answered correctly." This is true. What else might be the case if $5+7=13$ ? Plausibly, $5+6=12$. Conversely, if $5+6=12$, it would seem reasonable to expect that $5+7=13$. From (R6) and the truth of Fred's initial remark, we can infer "if $5+6=12$, George would have answered correctly," which is not obviously true. Repeated application of (R6) may help make the problem clearer:

1. $5+7=13 \square \rightarrow$ George answered correctly

2. $5+7=13 \square \rightarrow 5+8=14$

\footnotetext{
${ }^{10}$ The proof of (R6) is somewhat more convoluted than the proofs of the other inferences. It is useful to first prove the following lemma: if $\exists S \in \$_{w}$ such that $\emptyset \neq S \cap[\phi] \subseteq[\psi]$ and $\exists T \in \$_{w}$ such that $\emptyset \neq T \cap[\psi] \subseteq[\phi]$, then $\exists Z \in \$_{w}$ such that $\emptyset \neq Z \cap[\phi]=Z \cap[\psi]$. Suppose the antecedent and the negation of the consequent. Then $\emptyset \neq C \cap[\phi] \subseteq[\psi]$ and $\emptyset \neq D \cap[\psi] \subseteq[\phi] . \quad \forall Z \in \$_{w}(Z \cap[\phi] \neq Z \cap[\psi] \vee \emptyset=Z \cap[\phi])$ and $\emptyset \neq C \cap[\phi]$ imply $C \cap[\phi] \neq C \cap[\psi]$. Since $\emptyset \neq D \cap[\psi] \subseteq[\phi]$ implies $\emptyset \neq D \cap[\phi]$, by the same reasoning as above we get $D \cap[\phi] \neq D \cap[\psi]$. If $x \in C \cap[\phi]$ and $x \notin C \cap[\psi], x \in C \cap[\phi] \subseteq[\psi]$ implies $x \in C \cap[\psi]$ which is impossible; so $x \notin C \cap[\phi]$ and $x \in C \cap[\psi]$. Parallel reasoning shows that there is a $y$ such that $y \in D \cap[\phi]$ and $y \notin D \cap[\psi]$. By nesting, either $C \subseteq D$ or $D \subset C$. If $C \subseteq D$, then $x \in D \cap[\psi] \subseteq[\phi]$ implies $x \in C \cap[\phi]$ which is impossible. But if $D \subset C$, then $y \in C \cap[\phi] \subseteq[\psi]$ implies $y \in D \cap[\psi]$ which is impossible. This proves the lemma. Now consider an arbitrary world $w \in N$ of an arbitrary model such that $(\phi \square \leftrightarrow \psi) \rho_{w} 1$ and $(\psi \square \leftrightarrow \phi) \rho_{w} 1$. By the lemma, $\exists Z \in \$_{w}$ such that $\emptyset \neq Z \cap[\phi]=Z \cap[\psi]$. Then it is clear that $Z \cap[\phi] \subseteq[\chi]$ if and only if $Z \cap[\psi] \subseteq[\chi]$, which establishes (R6).

${ }^{11}$ This is a variation on an objection of Williamson's to non-vacuous truth conditions for counterpossibles discussed in [2, pp. 649-50].
}

Australasian Journal of Logic (14:4) 2017, Article no. 1 
3. $5+8=14 \square \mapsto 5+7=13$

4. $(5+7=13 \square \rightarrow$ George answered correctly $) \leftrightarrow(5+8=14 \square \leftrightarrow$ George answered correctly)

5. $5+8=14 \square \rightarrow 5+9=15$

6. $5+9=15 \square \rightarrow 5+8=14$

7. $(5+8=14 \square \rightarrow$ George answered correctly $) \leftrightarrow(5+9=15 \square \rightarrow$ George answered correctly)

8. ...

258. $(5+99=105 \square$ George answered correctly $) \leftrightarrow(5+100=106 \square \leftrightarrow$ George answered correctly)

259. $5+100=106 \square \rightarrow$ George answered correctly

What is going amiss in this chain of inferences? When we evaluate conditionals like (2), what is salient, given the parallel structure of antecedent and consequent, is minimizing the aberrance of impossible addition relative to actual (@) addition like so: $+(a, b)=a+@ b+@ k$. We restrict the impossible worlds we consider accordingly; since the antecedent worlds set $k=1$, the consequent holds at each of them. When we come to conditionals like (259), no such guide is offered and it is not clear what to minimize the aberrance of impossible addition to. Worlds where addition is just like actual addition except when one of the arguments is 100 have as much right to be among the closest impossible worlds as any other. But then, (259) is false.

An advocate for $S^{\#}$ might insist that conditionals like (2) are false. They would suggest that it is a mistake to not include worlds where addition works just like actual addition except when one of the arguments is 7 among the nearest impossible worlds. At such worlds, the consequent would not be true, so the whole conditional would fail. But this is just to beg the question against the intuitive truth of the pertinent conditional. Similarly, insisting on the truth of (259) when it seems intuitively false is pointless. One possible lesson to take away from this is that the worlds to be considered when evaluating a counterfactual or counterpossible might sometimes depend not only on the antecedent but on the consequent; how to spell this out without trivializing counterfactuals is an interesting project, but beyond the scope of this paper 12

\footnotetext{
${ }^{12}$ These issues are discussed by Gabbay in 9 .
} 
Since (R6) is characteristic of the basic sphere semantics I presented, I have treated the counterexample to (R6) as tantamount to a counterexample to sphere semantics. Given that there are a number of equivalent formulations of Lewis' semantics, this may be thought to be somewhat misleading; the same counterexample will apply to any selection function semantics in which it is required that $f_{\phi}(w) \subseteq[\psi]$ and $f_{\psi}(w) \subseteq[\phi]$ imply $f_{\phi}(w)=f_{\psi}(w) !^{13}$ On the other hand, a semantics using spheres might be contrived so as not to validate (R6). Put in this light, my objection is not so much to the technical apparatus of sphere semantics as to the notion of overall similarity it was explicitly developed to capture. It is implicit in this picture that the system of spheres about a world be invariant under consideration of different conditionals; the assignment of spheres depends only on the world. Given this picture, (R6) follows as a matter of course ${ }^{14}$

If this objection succeeds, $S^{\#}$ and all of its extensions fail. Additional objections were brought against $S_{2}^{\#}$ and $S_{3}^{\#}$. Even the advocate of a semantics as weak as $S^{\#}$ must contend with the claim that $\lambda$ is the closest impossible world, since the argument for that hinges only on intuitions about overall similarity which motivate each of the accounts. Therefore, sphere semantics is not the right approach for dealing with counterpossibles.

\section{The Alternative}

Another account of conditionals, with roots in [3], has been advocated for by Priest in [16]-[17]: the ceteris paribus account. The logics I present below expand on this work. As a preliminary, it should be noted that Priest intends for his preferred semantics to apply to conditionals generally, and not merely counterfactuals [16]. I do not take a stand on whether any of the logics developed here are adequate as an account of both indicative and subjunctive conditionals, or whether those are even the categories that we ought to theorize about conditionals in terms of. I do, however, contend that at least one of these systems meets certain adequacy conditions for an uniform treatment of counterfactuals and counterpossibles.

\subsection{Semantics}

The basic intuition underlying both Lewis' and Priest's accounts is the same: in evaluating a counterfactual, we look to some, but not all, of the worlds where the antecedent holds. Priest's suggestion is that "we look at those

\footnotetext{
${ }^{13}$ For alternative formulations and this condition, see [18], [1] and [14].

${ }^{14}$ For a discussion of some of these issues, see [7].
} 
[antecedent] worlds that are, ceteris paribus, the same as ours" [16, p. 329]. How this idea is formally captured will occupy the remainder of this section.

The logics I develop here are modifications of the logic Priest calls C [15, p. 85]. For this reason, I will call the most basic system $C^{\#}$. The language of $C^{\#}$ is the same as the language of the previous section, i.e. the language of propositional modal logic augmented with $\square \rightarrow$.

DEFINITION. An interpretation is a structure $\left\langle W, N,\left\{R_{\phi}: \phi \in \mathcal{F}\right\}, \rho\right\rangle$, where $W$ is a set of worlds and $\emptyset \neq N \subseteq W$ is the set of normal worlds. $\mathcal{F}$ is the set of $w f f s$ and $\left\{R_{\phi}: \phi \in \mathcal{F}\right\}$ is a set of subsets of $N \times W$, one for each $\phi \in \mathcal{F}$. $w R_{\phi} x$ is read: $x$ is ceteris paribus the same as $w$ with $\phi$ true (cf. [15, p. 85]). For all $w \in W, \rho_{w}$ is a relation between propositional letters and $\{0,1\}$. Moreover, for all $w \in W-N, \rho_{w}$ is a relation between formulae generally and $\{0,1\}$. Finally, for all propositional letters $\phi$ and $w \in N$, either $\phi \rho_{w} 1$ or $\phi \rho_{w} 0$, but not both. 1 is the designated value ("true"). Define $f_{\phi}(w)$ to be $\left\{x \in W: w R_{\phi} x\right\}$.

DEFINITION. Truth conditions for complex formulae at normal worlds $(w \in N)$ are exactly as in $S^{\#}$ and its extensions, except for the case involving counterfactuals:

7. $(\phi \square \rightarrow \psi) \rho_{w} 1$ if and only if $f_{\phi}(w) \subseteq[\psi] ; 0$ otherwise

Validity is defined as truth preservation at all normal worlds of all interpretations. It is how the notion of being "ceteris paribus the same as with $\phi$ true" is unpacked that determines the characteristic features of the logic. Formally speaking, what matters are what properties are imposed on $R_{\phi}$, and consequently on $f_{\phi}(w){ }^{15}$ Some conditions that might be imposed are the following:

$(\operatorname{Tru}) f_{\phi}(w) \subseteq[\phi]$

$\left(\mathrm{WC}^{*}\right)$ If $w \in[\phi], w \in f_{\phi}(w)$

$\left(\mathrm{SC}^{*}\right)$ If $w \in[\phi],\{w\}=f_{\phi}(w)$

(Dis*) If $N \cap[\phi] \neq \emptyset, f_{\phi}(w) \subseteq N$

\footnotetext{
${ }^{15}$ To develop the suggestion that the worlds to be considered in evaluating a counterfactual might depend on both the antecedent and consequent, substitute $\left\{R_{\phi, \psi}:(\phi, \psi) \in \mathcal{F}^{2}\right\}$ for $\left\{R_{\phi}: \phi \in \mathcal{F}\right\}$ in the definition of an interpretation. Now define $f_{\phi, \psi}(w)$ to be $\left\{x \in W: w R_{\phi, \psi} x\right\}$. Then $(\phi \square \leftrightarrow \psi) \rho_{w} 1$ if and only if $f_{\phi, \psi}(w) \subseteq[\psi]$. In this framework, it is possible for $(\phi \square \rightarrow \psi)$ and $(\phi \square \rightarrow \chi)$ to be evaluated using different worlds. While it is desirable to require that $f_{\phi, \psi}(w) \subseteq[\phi]$, requiring that $f_{\phi, \psi}(w) \subseteq[\psi]$ would obviously result in triviality.
}

Australasian Journal of Logic (14:4) 2017, Article no. 1 
The adoption of (Tru) and (WC*) yield a logic closely related to the system Priest calls $\mathrm{C}^{+}[15$, p. 88]. The arguments for adopting both conditions are straightforward. (Tru) is a direct consequence of the intuition that counterfactuals are evaluated by looking at worlds where the antecedent holds. The motivation for condition ( $\mathrm{WC}^{*}$ ) is that, if $w$ is an antecedent world, it is also such as to be ceteris paribus the same as itself with the antecedent true.

The arguments for ( $\mathrm{SC}^{*}$ ) and (Dis*) closely parallel the arguments for their relatives in sphere semantics. To say something briefly about (Dis*), the idea is that, given a possible antecedent, if some world is ceteris paribus the same as a given possible world with that possible antecedent holding, it should be a possible world. Again, some implicit appeal is apparently being made to the priority of logical laws in judgments of ceteris paribus similarity. For this reason, (Dis*) raises similar justificatory problems to (Dis), in addition to licensing similarly problematic inferences (but more on this anon).

Some extensions of $C^{\#}$ that are worthy of note are as follows $\left(C^{\#}-C_{3}^{\#}\right.$ are non-sphere versions of $S^{\#}-S_{3}^{\#}$ respectively): ${ }^{16}$

$$
\begin{aligned}
& C_{1}^{\#}: C^{\#}+(\mathrm{Tru})+\left(\mathrm{WC}^{*}\right) \\
& C_{2}^{\#}: C^{\#}+(\mathrm{Tru})+\left(\mathrm{SC}^{*}\right) \\
& C_{3}^{\#}: C^{\#}+(\mathrm{Tru})+\left(\mathrm{WC}^{*}\right)+\left(\mathrm{Dis}^{*}\right)
\end{aligned}
$$

Using model theoretic reasoning, it is easy to show, for example, $\diamond \phi, \square(\phi \rightarrow$ $\psi) \models_{C_{3}^{\#}} \phi \square \rightarrow \psi$. Assume that the premises are satisfied at an arbitrary world $w \in N$ of an arbitrary model, but that the conclusion is not. Then $\exists x \in f_{\phi}(w)$ such that $x \notin[\psi]$. By (Tru) and (Dis*), $x \in[\phi] \cap N$. But then $x \in[\psi]$, which is a contradiction.

To show that $\phi \square \mapsto \psi, \psi \square \hookrightarrow \phi \forall_{C_{3}^{\#}}(\phi \square \mapsto \chi) \leftrightarrow(\psi \square \mapsto \chi)$, we construct a counter-model. Let $W=N=f_{\phi}\left(w_{0}\right)=[\phi]=[\psi]=\left\{w_{0}, w_{1}\right\}, f_{\psi}\left(w_{0}\right)=$ $[\chi]=\left\{w_{0}\right\}$, and $f_{\phi}\left(w_{1}\right)=f_{\psi}\left(w_{1}\right)=\left\{w_{1}\right\}$. Then $(\phi \square \leftrightarrow \psi) \rho_{w_{0}} 1,(\psi \square \leftrightarrow$ $\phi) \rho_{w_{0}} 1$, and $(\psi \square \hookrightarrow \chi) \rho_{w_{0}} 1$, but $(\phi \square \mapsto \chi) \rho_{w_{0}} 0$. (R6) fails to be valid in all of the extensions of $C^{\#}$ considered here.

Sound and complete tableaux for $C^{\#}$ and its extensions are given in the appendix.

\footnotetext{
${ }^{16}$ It is not entirely clear what would correspond to $(\mathrm{Ab})$ in the $C^{\#}$ family, and it does little harm to leave it aside since $\lambda$ need not exist in all models.
} 


\subsection{Discussion}

The main point in favor of $C^{\#}$ and its extensions over $S^{\#}$ and its extensions is that none of the former, but all of the latter, validate (R6). If the objection against (R6) is persuasive and one of these approaches to giving a semantics for counterpossibles is correct, then the right semantics for counterpossibles must be found among the extensions of $C^{\#}$.

A second argument against sphere semantics concerns its underlying motivation. The idea of ordering worlds into nested spheres according to their overall similarity raises questions about how to judge overall similarity. If priority is given to logical laws, it seems that an argument can be made that the trivial world is among the closest impossible worlds to any given possible world. But this is clearly an undesirable outcome. However, this argument leaves the $C^{\#}$ advocate in a weaker dialectical position than the first since similar concerns can be raised about ceteris paribus sameness.

If the foregoing points favor $C^{\#}$ and its extensions, they do not determine which extension should be adopted. The inferences that I noted were problematic for $S_{2}^{\#}$ and $S_{3}^{\#}$ also hold in $C_{2}^{\#}$ and $C_{3}^{\#}$ respectively. Therefore, neither of those extensions should be adopted as a semantics for counterfactuals and counterpossibles.

I suggested that (R3)-(R5) are required of any minimally adequate semantics. $C^{\#}$ is too weak to validate these, but they hold in $C_{1}^{\#}$. Therefore, of the options presented in this paper, I believe that $C_{1}^{\#}$ offers the best semantics for counterfactuals and counterpossibles. Nonetheless, $C_{1}^{\#}$ is a relatively weak logic and logics intermediate between, or related to, $C_{1}^{\#}$ and $C_{3}^{\#}$ ought to be explored. That project is beyond the scope of this paper though 17

\section{Conclusion}

If it is granted that counterpossibles should be given non-vacuous truth conditions, a question remains about how exactly this should be done. One popular strategy is to develop modifications of Lewis' semantics with impossible worlds. A counterpossible can then fail when some impossible worlds where the antecedent is true but the consequent is not are closer to the actual world than any where both the antecedent and consequent are true.

Above, I developed several versions of Lewis-style sphere semantics and argued that they are all unsatisfactory. I showed how prima facie appeal-

\footnotetext{
${ }^{17}$ To give just a flavor, consider a variant on (Dis*), (WD): If $N \cap[\phi] \neq \emptyset, f_{\phi}(w) \cap N \neq \emptyset$. The result of adding (WD) to $C_{1}^{\#}$ can be called $C_{1.5}^{\#}$. This is a proper extension of $C_{1}^{\#}$ : $\diamond \phi, \square \psi \models_{C_{1.5}^{\#}} \neg(\phi \square \rightarrow \neg \psi)$. Note that this inference also fails in $C_{3}^{\#}$.
} 
ing assumptions like (Dis) lead to special justificatory problems and license intuitively invalid inferences. I contended that all extensions of $S^{\#}$ sanction at least one undesirable inference. Thus, I argued that this approach is mistaken.

I then proposed and developed an alternative approach. $C^{\#}$ and its extensions, which take the notion of being ceteris paribus the same with the antecedent true as basic, form a family of plausible logics for counterfactuals and counterpossibles. Some of the extensions of $C^{\#}$ were shown to be subject to similar problems as certain extensions of $S^{\#}$. Nevertheless, at least one extension- $C_{1}^{\#}$-met what I suggested were minimum adequacy conditions for a semantics without validating any of the problematic inferences considered above. To close on a programmatic note, I believe that fruitful work might follow by examining logics related to $C_{1}^{\#}$ and $C_{3}^{\#} \cdot 18$

\section{A Tableaux for $C^{\#}$ and Extensions}

A tree is a structure such as the following:

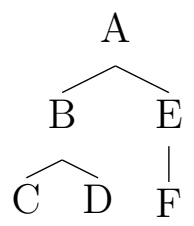

A, B and so on are nodes. Nodes have one of the following forms: $N(i)$, $i r_{\phi} j, i=j$ and $\phi, \pm i$. The node $\mathrm{A}$ is the root of the tree. A branch is a maximal path, e.g. $\{\mathrm{A}, \mathrm{E}, \mathrm{F}\}$. A branch is closed if it contains nodes of the form $\phi,+i$ and $\phi,-i$. A tableau is closed if each branch is closed. A tableau is complete if every applicable rule has been applied. Where $\Sigma$ is a (finite) set of formulae, $\Sigma \vdash \phi$ if and only if there is a closed tableau whose initial list consists of nodes of the form $\psi,+0$ for all $\psi \in \Sigma, \phi,-0$ and $N(0)$. The tableaux rules are as follows:

1. Extensional Rules (for any branch containing the first two nodes, it can be extended in any of the following ways):

\footnotetext{
${ }^{18}$ I am grateful to Graham Priest and the members of the Logic and Metaphysics Workshop of the Graduate Center of the City University of New York for their comments on earlier versions of this paper. I am also grateful to the anonymous referees of earlier versions of this paper for suggestions that led to its improvement.
} 


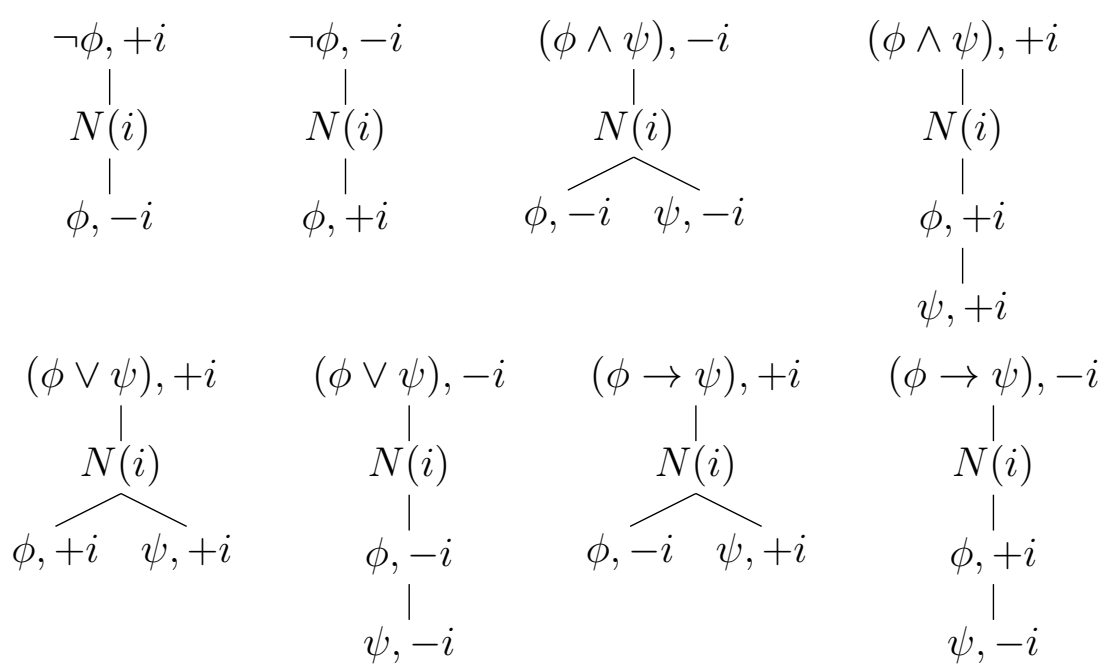

2. Intensional Rules I (for any branch containing the first two nodes and $j$ new):
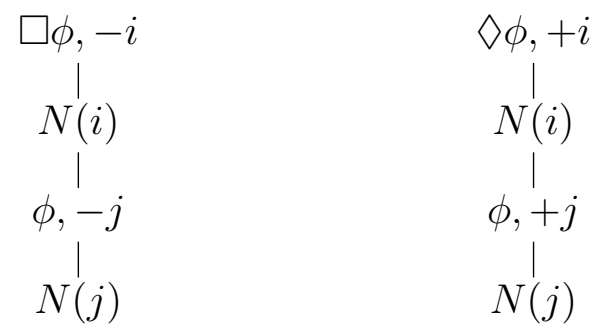

3. Intensional Rules II (for any branch containing the first three nodes, or first two where $i=j$ ):
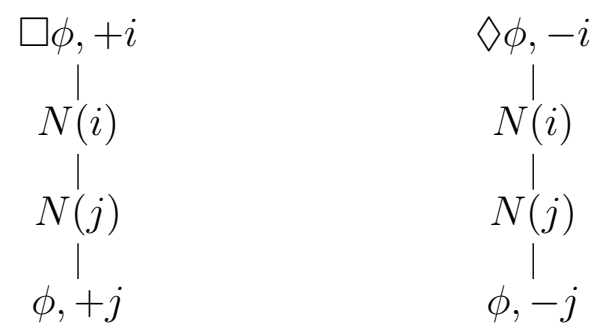

4. $C^{\#}$ Rules (in addition to $1-3$ ):

(a) Positive Rule (for any branch containing the first three nodes):

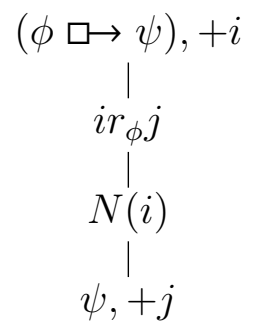

Australasian Journal of Logic (14:4) 2017, Article no. 1 
(b) Negative Rule (for any branch containing the first two nodes and $j$ new):

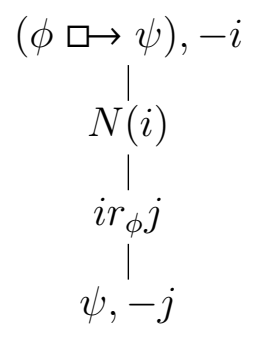

5. $C_{1}^{\#}$ Rules (in addition to $\left.1-3,4 a\right)$ :

(a) Weak Centering Rule (for any branch containing the first node and antecedents $\phi$ of counterfactuals on the branch) 19

$$
\overbrace{\substack{i r_{\phi} i \\ \phi i}}^{N(i)}-i
$$

(b) Negative Rule (for any branch containing the first two nodes and $j$ new):

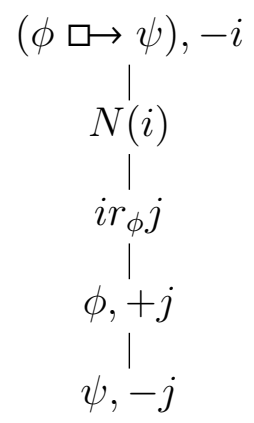

6. $C_{2}^{\#}$ Rules (in addition to $\left.1-3,4 \mathrm{a}, 5\right)$ :

(a) Strong Centering Rule (for any branch containing the first three nodes):

\footnotetext{
${ }^{19}$ At a presentation of this work, it was asked whether this rule could be replaced with modus ponens. The tableaux which result from such a replacement are sound, but not complete.
}

Australasian Journal of Logic (14:4) 2017, Article no. 1 


$$
\begin{gathered}
\phi,+i \\
\vdots r_{\phi} j \\
\vdots \\
N(i) \\
i=j
\end{gathered}
$$

(b) Identity Rules (the reflexivity rule is applied for all old $i$; the other rule is applied for any branch containing the first two nodes where $\alpha(j)$ is an arbitrary $j$-containing node and $\alpha(i / j)$ is the result of replacing some occurrences of $j$ by $i$ in $\alpha$ ):

$$
\begin{array}{cc}
i & i=j \\
i=i & \alpha(j) \\
& \alpha(i / j)
\end{array}
$$

7. $C_{3}^{\#}$ Rules (in addition to $1-3,4 \mathrm{a}, 5$; for any branch containing the first two nodes):

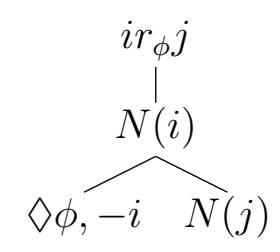

Example. $\diamond \phi, \square(\phi \rightarrow \psi) \vdash_{C_{3}^{\#}}(\phi \square \mapsto \psi)$ 


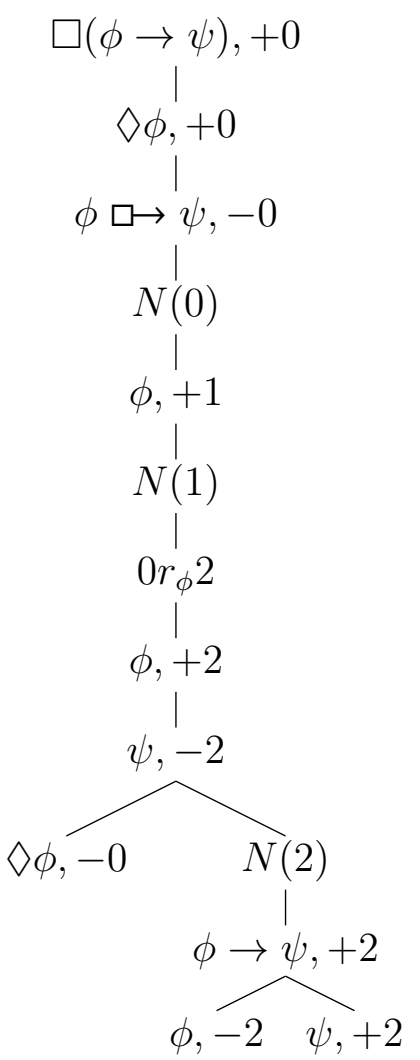

I now prove soundness and completeness for $C^{\#}$ and demonstrate how to extend the proofs for $C_{1}^{\#}-C_{3}^{\#}$. The proofs and definitions of this section generally follow the paradigm of those in [15].

DEFINITION. Let $\mathcal{I}=\left\langle W, N,\left\{R_{\phi}: \phi \in \mathcal{F}\right\}, \rho\right\rangle$ be a $C^{\#}$ interpretation and $\mathrm{b}$ be a branch of a tableau. $\mathcal{I}$ is faithful to $\mathrm{b}$ if and only if there is a function $g: \mathbb{Z} \rightarrow W$ such that: ${ }^{20}$

1. If $\phi,+i$ is on $\mathrm{b}, \phi \rho_{g(i)} 1$ in $\mathcal{I}$

2. If $\phi,-i$ is on b, not $\phi \rho_{g(i)} 1$ in $\mathcal{I}$

3. If $N(i)$ is on b, $g(i) \in N$ in $\mathcal{I}$

4. If $i r_{\phi} j$ is on b, $g(i) R_{\phi} g(j)$ in $\mathcal{I}$ (i.e. $g(j) \in f_{\phi}(g(i))$ )

LEMMA 1. If $\mathcal{I}$ is faithful to $b$ and $a C^{\#}$ tableau rule is applied to $b$, then $\mathcal{I}$ is faithful to at least one of the resulting branches $b^{*}$

${ }^{20} \mathbb{Z}$ is $\{0,1,2, \ldots\}$.

Australasian Journal of Logic (14:4) 2017, Article no. 1 
Proof. The proof is by cases of the rule applied. This is fairly routine, so I will only examine the rules which pertain to intensional formulae.

Suppose that $\mathcal{I}$ is faithful to b and $\square \phi,-i$ and $N(i)$ occur on b. Rule (2) is applied and $\mathrm{b}$ is extended to a branch $\mathrm{b}^{*}$ with nodes $\phi,-j$ and $N(j)$ (for $j$ new). By the faithfulness of $\mathcal{I}$, since $g(i) \in N$ and not $\square \phi \rho_{g(i)} 1, \square \phi \rho_{g(i)} 0$ in $\mathcal{I}$. Hence $\exists w \in N$ such that $w \notin[\phi]$. Then if $h$ is the same as $g$ except that $h(j)=w, h$ shows that $\mathcal{I}$ is faithful to $\mathrm{b}^{*}$. The case with $\nabla \phi,+i$ is the same.

Suppose that $\mathcal{I}$ is faithful to $\mathrm{b}$ and $\square \phi,+i, N(i)$ and $N(j)$ occur on b. Rule (3) is applied and $\mathrm{b}$ is extended to a branch $\mathrm{b}^{*}$ with node $\phi,+j$. By the faithfulness of $\mathcal{I}, \square \phi \rho_{g(i)} 1, g(j) \in N$ and $g(i) \in N$. Then $g(j) \in N \subseteq[\phi]$ and $\mathcal{I}$ is faithful to $\mathrm{b}^{*}$. The case with $\diamond \phi,-i$ is the same.

Suppose that $\mathcal{I}$ is faithful to $\mathrm{b}$ and $(\phi \square \rightarrow \psi),+i, i r_{\phi} j$ and $N(i)$ occur on b. Rule (4a) is applied and b is extended to a branch $\mathrm{b}^{*}$ with node $\psi,+j$. From the faithfulness of $\mathcal{I},(\phi \square \rightarrow \psi) \rho_{g(i)} 1, g(j) \in f_{\phi}(g(i))$ and $g(i) \in N$ in $\mathcal{I}$. Because $(\phi \square \leftrightarrow \psi) \rho_{g(i)} 1, f_{\phi}(g(i)) \subseteq[\psi]$, so $g(j) \in[\psi]$. Therefore, $\mathcal{I}$ is faithful to $b^{*}$.

Finally, suppose that $\mathcal{I}$ is faithful to $\mathrm{b}$ and that $(\phi \square \leftrightarrow \psi),-i$ and $N(i)$ occur on $\mathrm{b}$. Then rule (4b) is applied and $\mathrm{b}$ is extended to a branch $\mathrm{b}^{*}$ with nodes $i r_{\phi} j$ and $\psi,-j$ (for $j$ new). Since $\mathcal{I}$ is faithful to b, $g(i) \in N$ but not $(\phi \square \rightarrow \psi) \rho_{g(i)} 1$ in $\mathcal{I}$; that is, $(\phi \square \rightarrow \psi) \rho_{g(i)} 0$ in $\mathcal{I}$. Hence $\exists w \in f_{\phi}(g(i))$ such that $w \notin[\psi]$. Then if $h$ is the same as $g$ except that $h(j)=w, h$ shows that $\mathcal{I}$ is faithful to $b^{*}$.

THEOREM 1 (Soundness). If $\Sigma \vdash_{C \#} \phi, \Sigma \models_{C \#} \phi$

Proof. Suppose that $\Sigma \vdash \phi$ but it is not the case that $\Sigma \models \phi$. Then there is an interpretation $\mathcal{I}$ such that $\exists w \in N$ for which $\forall \chi \in \Sigma, \chi \rho_{w} 1$ and $\phi \rho_{w} 0$. As $\Sigma \vdash \phi$, there is a closed tableau $\mathrm{T}$ and $\mathcal{I}$ is faithful to the initial segment of $\mathrm{T}$ (i.e. the nodes antecedent to rule applications) because of the function $g(0)=w$. By repeated application of Lemma 1 , there is a branch $\mathrm{b}$ of $\mathrm{T}$ such that $\mathcal{I}$ is faithful to each segment of $\mathrm{b}$. If $\mathrm{T}$ is closed, however, $\mathrm{b}$ must be as well; then $\mathrm{b}$ has nodes of the form $\psi,+k$ and $\psi,-k$. This is a contradiction, since by faithfulness, both $\psi \rho_{g(k)} 1$ and not $\psi \rho_{g(k)} 1$ in $\mathcal{I}$.

DEFINITION. The interpretation $\mathcal{I}=\left\langle W, N,\left\{R_{\phi}: \phi \in \mathcal{F}\right\}, \rho\right\rangle$ induced by an open branch $b$ is such that:

1. $W=\left\{w_{i}: i\right.$ on $\left.\mathrm{b}\right\}$

2. $N=\left\{w_{i}: N(i)\right.$ on $\left.\mathrm{b}\right\}$

3. For counterfactual antecedents (on b) $\phi, w_{i} R_{\phi} w_{j}$ if and only if $i r_{\phi} j$ on $\mathrm{b}$ 
4. For $w_{i} \in W-N, \phi \rho_{w_{i}} 1$ if and only if $\phi,+i$ on $\mathrm{b}$

5. For $w_{i} \in W-N$, not $\phi \rho_{w_{i}} 1$ if and only if $\phi,-i$ on $\mathrm{b}$

6. For propositional letters $\psi$ and $w_{i} \in N, \psi \rho_{w_{i}} 1$ if and only if $\psi,+i$ on $\mathrm{b}$

7. For propositional letters $\psi$ and $w_{i} \in N, \psi \rho_{w_{i}} 0$ if and only if $\psi,-i$ on $\mathrm{b}$

LEMMA 2 (Truth Lemma). If $b$ is an open, complete branch of a tableau and $\mathcal{I}$ is an interpretation induced by $b$, then:

1. If $\phi,+i$ occurs on $\mathrm{b}, \phi \rho_{w_{i}} 1$ in $\mathcal{I}$

2. If $\phi,-i$ occurs on $\mathrm{b}$, not $\phi \rho_{w_{i}} 1$ in $\mathcal{I}$

Proof. Proof is by induction on the complexity of $\phi$. Once again, I will only examine the cases which pertain to intensional formulae.

Suppose that $\phi$ is of the form $\square \psi$ and $\phi,+i$ is on b. Then if $N(i)$ does not occur on $b$, the result holds by definition. Alternatively, if it does, then for every $j$ such that $N(j)$ occurs on $\mathrm{b}, \psi,+j$ is on $\mathrm{b}$ since $\mathrm{b}$ is complete and rule (3) has been applied. By the induction hypothesis, $N \subseteq[\psi]$, which implies $\phi \rho_{w_{i}} 1$ in $\mathcal{I}$. Suppose instead that $\phi,-i$ is on b. If $N(i)$ does not occur on b, the result holds by definition. If it does, then there is a $j$ such that $\psi,-j$ and $N(j)$ occur on b. By the induction hypothesis, $w_{j} \in N$ but $w_{j} \notin[\psi]$, so $\phi \rho_{w_{i}} 0$, which implies not $\phi \rho_{w_{i}} 1$ in $\mathcal{I}$. The case where $\phi$ is of the form $\diamond \psi$ is the same.

Suppose that $\phi$ is of the form $(\theta \square \mapsto \psi)$ and $\phi,+i$ is on b. Then if $N(i)$ does not occur on $b$, the result holds by definition. Alternatively, if it does, then for every $j$ such that $i r_{\theta} j$ is on $\mathrm{b}, \psi,+j$ is on $\mathrm{b}$ since $\mathrm{b}$ is complete and rule (4a) has been applied. By the induction hypothesis, $f_{\theta}\left(w_{i}\right) \subseteq[\psi]$, which implies $\phi \rho_{w_{i}} 1$ in $\mathcal{I}$. Suppose instead that $\phi,-i$ is on b. If $N(i)$ does not occur on $\mathrm{b}$, the result holds by definition. If it does, then there is a $j$ such that $i r_{\theta} j$ and $\psi,-j$ each occur on b. By the induction hypothesis, $w_{j} \in f_{\theta}\left(w_{i}\right)$ but $w_{j} \notin[\psi]$. So $\phi \rho_{w_{i}} 0$, which implies not $\phi \rho_{w_{i}} 1$ in $\mathcal{I}$.

THEOREM 2 (Completeness). If $\Sigma \models_{C \#} \phi, \Sigma \vdash_{C \#} \phi$

Proof. Suppose that it is not the case that $\Sigma \vdash \phi$. Then the attempted proof results in a completed open tableau with at least one complete open branch b. Let $\mathcal{I}$ be an interpretation induced by b. Then in $\mathcal{I}, \forall \psi \in \Sigma, \psi \rho_{w_{0}} 1$ but $\phi \rho_{w_{0}} 0$ (by Lemma 2). So it is not the case that $\Sigma \models \phi$.

To prove the soundness of $C_{1}^{\#}$, the definition of faithfulness is the same except that the interpretation is now a $C_{1}^{\#}$ interpretation. 
LEMMA 3. If $\mathcal{I}$ is faithful to $b$ and $a C_{1}^{\#}$ tableau rule is applied to $b$, then $\mathcal{I}$ is faithful to at least one of the resulting branches $b^{*}$

Proof. I examine only the two cases that differ from $C^{\#}$.

Suppose that $\mathcal{I}$ is faithful to $\mathrm{b}$ and $N(i)$ appears on b. Rule (5a) is applied which extends b to $\mathrm{b}^{*}$ with $\phi,+i$ and $i r_{\phi} i$ or $\mathrm{b}^{*}$ with $\phi,-i$. In $\mathcal{I}, g(i) \in N$. By $\left(\mathrm{WC}^{*}\right)$, either $\phi \rho_{g(i)} 0$ or if $1, g(i) \in f_{\phi}(g(i))$. In the first case, $\mathcal{I}$ is faithful to $\mathrm{b}^{*}$ with $\phi,-i$; in the second, it is faithful to $\mathrm{b}^{*}$ with $\phi,+i$ and $i r_{\phi} i$.

Suppose that $\mathcal{I}$ is faithful to b and that $(\phi \square \rightarrow \psi),-i$ and $N(i)$ occur on $\mathrm{b}$. Then rule $(5 \mathrm{~b})$ is applied and $\mathrm{b}$ is extended to a branch $\mathrm{b}^{*}$ with nodes $i r_{\phi} j, \phi,+j$ and $\psi,-j$ (for $j$ new). Since $\mathcal{I}$ is faithful to b, $g(i) \in N$ but not $(\phi \square \hookrightarrow \psi) \rho_{g(i)} 1$ in $\mathcal{I}$; that is, $(\phi \square \rightarrow \psi) \rho_{g(i)} 0$ in $\mathcal{I}$. Hence $\exists w \in f_{\phi}(g(i))$ (and by (Tru), also in $[\phi])$ such that $w \notin[\psi]$. Then if $h$ is the same as $g$ except that $h(j)=w, h$ shows that $\mathcal{I}$ is faithful to $\mathrm{b}^{*}$.

THEOREM 3 (Soundness). If $\Sigma \vdash_{C_{1}^{\#}} \phi, \Sigma \models_{C_{1}^{\#}} \phi$

Proof. The proof proceeds exactly as in the case of $C^{\#}$.

For the proof of the completeness of $C_{1}^{\#}$, the third condition in the definition of the interpretation induced by an open branch $b$ is modified as follows:

3. For counterfactual antecedents (on b) $\phi, w_{i} R_{\phi} w_{j}$ if and only if $i r_{\phi} j$ on b; otherwise, $w_{i} R_{\phi} w_{j}$ if and only if $w_{j} \in[\phi]$

The proof of the Truth Lemma proceeds almost exactly as before (there is only one insignificant change: if $\theta \square \leftrightarrow \psi,-i$ and $N(i)$ are on $\mathrm{b}$, then there is a $j$ such that $i r_{\theta} j, \theta,+j$ and $\psi,-j$ all occur on b). It must, however, be verified that the induced interpretation is indeed a $C_{1}^{\#}$ interpretation.

LEMMA 4. If $b$ is an open, complete branch of a tableau and $\mathcal{I}$ is an interpretation induced by $b, \mathcal{I}$ satisfies the $C_{1}^{\#}$ constraints on $f_{\phi}(w)$

Proof. There are two constraints to consider: (Tru) and (WC*). If $\phi$ does not occur as an antecedent, the constraints are satisfied by the definition of $R_{\phi}$ in $\mathcal{I}$. Otherwise, each of the two cases must be considered individually. Suppose that $w_{i} R_{\phi} w_{j}$ in $\mathcal{I}$. Then by the definition of $\mathcal{I}, i r_{\phi} j$ occurs on b. This node can occur only as a result of the application of one of two rules $(5 \mathrm{a}, \mathrm{b})$, each of which also introduce $\phi,+j$. By the Truth Lemma, $\phi \rho_{w_{j}} 1$ in $\mathcal{I}$.Thus, $f_{\phi}\left(w_{i}\right) \subseteq[\phi]$. Suppose that $w_{i} \in N$ and $\phi \rho_{w_{i}} 1$. Then $N(i)$ occurs on $\mathrm{b}$ and so do either $\phi,+i$ and $i r_{\phi} i$ or $\phi,-i$ by (5a). The Truth Lemma rules out the latter, so by the definition of the induced interpretation, $w_{i} \in f_{\phi}\left(w_{i}\right)$. 
THEOREM 4 (Completeness). If $\Sigma \models_{C_{1}^{\#}} \phi, \Sigma \vdash_{C_{1}^{\#}} \phi$

Proof. The proof proceeds exactly as in the case of $C^{\#}$.

Proving the soundness of $C_{2}^{\#}$ requires the addition of another condition to the definition of faithfulness:

5. If $i=j$ is on b, $g(i)=g(j)$ in $\mathcal{I}$

LEMMA 5. If $\mathcal{I}$ is faithful to $b$ and $a C_{2}^{\#}$ tableau rule is applied to $b$, then $\mathcal{I}$ is faithful to at least one of the resulting branches $b^{*}$

Proof. I examine the cases that differ from $C_{1}^{\#}$.

Suppose that $\mathcal{I}$ is faithful to $\mathrm{b}$ and $\phi,+i, i r_{\phi} j$ and $N(i)$ occur on b. Rule (6a) is applied and $\mathrm{b}$ is extended to a branch $\mathrm{b}^{*}$ with node $i=j$. By the faithfulness of $\mathcal{I}, \phi \rho_{g(i)} 1, g(j) \in f_{\phi}(g(i))$ and $g(i) \in N$. By (SC*), since $g(i) \in[\phi],\{g(i)\}=f_{\phi}(g(i))$ implying that $g(i)=g(j)$. Therefore, $\mathcal{I}$ is faithful to $\mathrm{b}^{*}$.

Suppose that $\mathcal{I}$ is faithful to $\mathrm{b}, i$ occurs at some node on $\mathrm{b}$ and the reflexivity rule is applied extending $\mathrm{b}$ to $\mathrm{b}^{*}$ with node $i=i$. Since $i$ is on $\mathrm{b}$, clearly $g(i)=g(i)$ in $\mathcal{I}$. Therefore, $\mathcal{I}$ is faithful to $\mathrm{b}^{*}$.

Lastly, suppose that $\mathcal{I}$ is faithful to $\mathrm{b}, i=j$ and $\alpha(j)$ occur on $\mathrm{b}$ and the substitution rule is applied which extends $\mathrm{b}$ to $\mathrm{b}^{*}$ with $\alpha(i / j)$. Then $g(i)=g(j)$ in $\mathcal{I}$. It is easily verified that $\mathcal{I}$ is faithful to $\mathrm{b}^{*}$ for each case of $\alpha$. For example, if $\alpha(j)$ is $k r_{\phi} j$ and $\alpha(i / j)$ is $k r_{\phi} i, g(j) \in f_{\phi}(g(k))$ and so $g(i) \in f_{\phi}(g(k))$ in $\mathcal{I}$, which implies that $\mathcal{I}$ is faithful to $\mathrm{b}^{*}$.

THEOREM 5 (Soundness). If $\Sigma \vdash_{C_{2}^{\#}} \phi, \Sigma \models_{C_{2}^{\#}} \phi$

Proof. The proof proceeds exactly as in the case of $C^{\#}$.

The proof of the completeness of $C_{2}^{\#}$ requires two key modifications to the definition of an interpretation induced by an open branch. First, I introduce some additional notation.

DEFINITION. $x \sim y$ if and only if $x=y$ occurs on tableau branch $\mathrm{b}$

Remark. $\sim$ is an equivalence relation. Let $\|x\|=\{y: x \sim y\}$.

In the definition of the induced interpretation, all subscripts are now equivalence classes. Moreover, the third condition is modified as follows:

3. For counterfactual antecedents (on b) $\phi, w_{\|i\|} R_{\phi} w_{\|j\|}$ if and only if $i r_{\phi} j$ on b; otherwise, $w_{\|i\|} R_{\phi} w_{\|j\|}$ if and only if $w_{\|j\|} \in\left\{w_{\|i\|}\right\} \cap[\phi]$ 
The proof of the Truth Lemma proceeds as in the case of $C^{\#}$ except the subscripts are equivalence classes.

LEMMA 6. If $b$ is an open, complete branch of a tableau and $\mathcal{I}$ is an interpretation induced by $b, \mathcal{I}$ satisfies the $C_{2}^{\#}$ constraints on $f_{\phi}(w)$

Proof. There are two constraints to consider, one of which-(Tru)-was already verified in the case of $C_{1}^{\#}$. If $\phi$ does not occur as an antecedent, $\left(\mathrm{SC}^{*}\right)$ is satisfied by the definition of $R_{\phi}$ in $\mathcal{I}$. Otherwise, suppose that $w_{\|i\|} \in$ $N, \phi \rho_{w_{\|i\|}} 1$ and $w_{\|i\|} R_{\phi} w_{\|j\|}$. Then $N(i)$ and $i r_{\phi} j$ occur on b, as do either $\phi,+i$ and $i r_{\phi} i$ or $\phi,-i$ by (5a). The Truth Lemma rules out the latter. But if $N(i), i r_{\phi} j$ and $\phi,+i$ occur on $\mathrm{b}$, so does $i=j$ by (6a). Then $w_{\|i\|} \in f_{\phi}\left(w_{\|i\|}\right)$ and, since $i \sim j, w_{\|i\|}=w_{\|j\|}$.

THEOREM 6 (Completeness). If $\Sigma \models_{C_{2}^{\#}} \phi, \Sigma \vdash_{C_{2}^{\#}} \phi$

Proof. The proof proceeds as in the case of $C^{\#}$ except the subscripts are equivalence classes.

To prove the soundness of $C_{3}^{\#}$, the definition of faithfulness is the same as in the case of $C^{\#}$ except that the interpretation is a $C_{3}^{\#}$ interpretation.

LEMMA 7. If $\mathcal{I}$ is faithful to $b$ and $a C_{3}^{\#}$ tableau rule is applied to $b$, then $\mathcal{I}$ is faithful to at least one of the resulting branches $b^{*}$

Proof. Only one rule differs from the case of $C_{1}^{\#}$.

Suppose that $\mathcal{I}$ is faithful to b and $N(i)$ and $i r_{\phi} j$ occur on b. Rule (7) is applied and $\mathrm{b}$ is extended to a branch $\mathrm{b}^{*}$ that either contains a node $\nabla \phi,-i$ or $N(j)$. In $\mathcal{I}, g(i) \in N$ and $g(j) \in f_{\phi}(g(i))$. From (Dis*), either $\forall w \in N, \phi \rho_{w} 0$; or if $w \in f_{\phi}(g(i)), w \in N$. In the first case, $\mathcal{I}$ is faithful to $\mathrm{b}^{*}$ with $\nabla \phi,-i$; in the second, it is faithful to $\mathrm{b}^{*}$ with $N(j)$.

THEOREM 7 (Soundness). If $\Sigma \vdash_{C_{3}^{\#}} \phi, \Sigma \models_{C_{3}^{\#}} \phi$

Proof. The proof proceeds exactly as in the case of $C^{\#}$.

For the proof of the completeness of $C_{3}^{\#}$, the third condition in the $C^{\#}$ definition of the interpretation induced by an open branch $b$ is modified as follows:

3. For counterfactual antecedents (on b) $\phi, w_{i} R_{\phi} w_{j}$ if and only if $i r_{\phi} j$ on b; otherwise, $w_{i} R_{\phi} w_{j}$ if and only if $w_{j} \in N \cap[\phi]$

The proof of the Truth Lemma proceeds just as in the case of $C^{\#}$. 
LEMMA 8. If $b$ is an open, complete branch of a tableau and $\mathcal{I}$ is an interpretation induced by $b, \mathcal{I}$ satisfies the $C_{3}^{\#}$ constraints on $f_{\phi}(w)$

Proof. There are three constraints to consider, but two of them are just those of $C_{1}^{\#}$. If $\phi$ does not occur as an antecedent, (Dis) is satisfied by the definition of $R_{\phi}$ in $\mathcal{I}$. Otherwise, suppose that $w_{i} \in N, \exists w_{k} \in N \cap[\phi]$ and $w_{i} R_{\phi} w_{j}$. Then $N(i)$ and $i r_{\phi} j$ occur on b, and so do either $\diamond \phi,-i$ or $N(j)$ by (7). By the Truth Lemma, it cannot be the first; so it must be that $f_{\phi}\left(w_{i}\right) \subseteq N$.

THEOREM 8 (Completeness). If $\Sigma \models_{C_{3}^{\#}} \phi, \Sigma \vdash_{C_{3}^{\#}} \phi$

Proof. The proof proceeds exactly as in the case of $C^{\#}$.

\section{References}

[1] Bjerring, J. (2014). On Counterpossibles. Philosophical Studies, 168 (2), 327-353.

[2] Brogaard, B., \& Salerno, J. (2013). Remarks on Counterpossibles. Synthese, 190 (4), 639-660.

[3] Chellas, B. (1975). Basic Conditional Logic. Journal of Philosophical Logic, 4 (2), 133-153.

[4] Chellas, B. (1980). Modal Logic: An Introduction. Cambridge: Cambridge University Press.

[5] DeRose, K. (1994). Lewis on 'Might' and 'Would' Counterfactual Conditionals. Canadian Journal of Philosophy, 24 (3), 413-418.

[6] DeRose, K. (1999). Can It Be That It Would Have Been Even Though It Might Not Have Been? Philosophical Perspectives, 13, 385-413.

[7] Djordjevic, V. (2013). Similarity and Cotenability. Synthese, 190 (4), 681691.

[8] Fine, K. (1975). Critical Notice of Lewis, Counterfactuals. Mind, 84 (335), 451-458.

[9] Gabbay, D. (1972). A General Theory of the Conditional in Terms of a Ternary Operator. Theoria, 38 (3), 97-104.

[10] Jago, M. (2015). Impossible Worlds. Noûs, 49 (4), 713-728.

Australasian Journal of Logic (14:4) 2017, Article no. 1 
[11] Lewis, D. (1973). Counterfactuals. Oxford: Oxford University Press.

[12] Lewis, D. (1981). Ordering Semantics and Premise Semantics for Counterfactuals. Journal of Philosophical Logic, 10 (2), 217-234.

[13] Nolan, D. (1997). Impossible Worlds: A Modest Approach. Notre Dame Journal of Formal Logic, 38 (4), 535-572.

[14] Nute, D. (1980). Topics in Conditional Logic. Dordrecht: D. Reidel Publishing Company.

[15] Priest, G. (2008). An Introduction to Non-Classical Logic (2nd ed.). Cambridge: Cambridge University Press.

[16] Priest, G. (2009). Conditionals: A Debate with Jackson. In I. Ravenscroft (Ed.), Minds, Ethics, and Conditionals: Themes from the Philosophy of Frank Jackson (pp. 311-336). Oxford: Oxford University Press.

[17] Priest, G. (2017). Some New Thoughts on Conditionals. Topoi, to appear

[18] Stalnaker, R. (1968). A Theory of Conditionals. In W. L. Harper, R. Stalnaker \& G. Pearce (Eds.), Ifs (pp. 41-55). Boston: D. Reidel Publishing Company.

[19] Yagisawa, T. (1988). Beyond Possible Worlds. Philosophical Studies, 53 (2), 175-204. 